\title{
The Uniqueness of Strong Solutions for the Camassa-Holm Equation
}

\author{
Meng $W u^{1}$ and Chong Lai $^{2}$ \\ ${ }^{1}$ The School of Mathematics, Southwestern University of Finance and Economics, Chengdu 610074, China \\ ${ }^{2}$ The School of Finance, Southwestern University of Finance and Economics, Chengdu 610074, China \\ Correspondence should be addressed to Meng Wu; wumeng@swufe.edu.cn
}

Received 28 February 2013; Accepted 10 May 2013

Academic Editor: Janusz Matkowski

Copyright (c) $2013 \mathrm{M}$. Wu and C. Lai. This is an open access article distributed under the Creative Commons Attribution License, which permits unrestricted use, distribution, and reproduction in any medium, provided the original work is properly cited. \begin{abstract}
Assume that there exists a strong solution of the Camassa-Holm equation and the initial value of the solution belongs to the Sobolev
\end{abstract} space $H^{1}(R)$. We provide a new proof of the uniqueness of the strong solution for the equation.

\section{Introduction}

The integrable Camassa-Holm model [1]

$$
u_{t}-u_{t x x}+3 u u_{x}=2 u_{x} u_{x x}+u u_{x x x}
$$

has been investigated by many scholars. Equation (1) has peaked solitary wave solutions, which takes the form $c e^{-|x-c t|}$, $c \in R$. The existence and uniqueness of the global weak solutions for (1) have been given by Constantin and Escher [2] and Constantin and Molinet [3] in which the $m=u_{0}-u_{0 x x}$ is a positive (or negative) Radon measure. The local wellposedness of strong solutions for the Camassa-Holm model and its various generalized forms are provided in [4-8]. For the initial value $u_{0}$ satisfying $u_{0}-u_{0 x x} \geq 0$ or $u_{0}-u_{0 x x} \leq$ 0 , it is shown in [9] that the Camassa-Holm equation has unique global strong solutions in the Sobolev space $H^{s}(R)$ with $s>3 / 2$. If the initial data satisfy certain conditions, we know that the local strong solutions blow up in finite time $[10,11]$. It means that the slope of the solution becomes unbounded while the solution itself remains bounded. For other techniques to obtain the dynamic properties for the Camassa-Holm equation and other related shallow water equations, the reader is referred to [12-16] and the references therein.
We consider the equivalent form of the Cauchy problem for (1)

$$
\begin{gathered}
u_{t}+\frac{1}{2}\left(u^{2}\right)_{x}+\partial_{x} P_{u}(t, x)=0, \\
u(0, x)=u_{0},
\end{gathered}
$$

where $P_{u}(t, x)=\Lambda^{-2}\left(u^{2}+(1 / 2) u_{x}^{2}\right)=(1 / 2) \int_{-\infty}^{\infty} e^{|x-y|}\left(u^{2}(t, y)+\right.$ $\left.(1 / 2) u_{y}^{2}(t, y)\right) d y$. If (1) has a suitable smooth strong solution, we have the conservation law

$$
\begin{aligned}
& \int_{-\infty}^{\infty}\left(u^{2}(t, x)+u_{x}^{2}(t, x)\right) d x \\
& \quad=\int_{-\infty}^{\infty}\left(u^{2}(0, x)+u_{x}^{2}(0, x)\right) d x
\end{aligned}
$$

which derives

$$
\|u\|_{L^{\infty}(R)} \leq\|u\|_{H^{1}(R)}=\left\|u_{0}\right\|_{H^{1}(R)} .
$$

The objective of this work is to give a new proof of the uniqueness for the solutions of the Camassa-Holm equation (1). Firstly, we establish the following inequality:

$$
\begin{aligned}
& \|u(t, \cdot)-v(t, \cdot)\|_{L^{1}(R)} \\
& \quad \leq c e^{c t}\left(\int_{-\infty}^{\infty}\left|u_{0}(x)-v_{0}(x)\right| d x+\int_{-\infty}^{\infty}\left|u_{0 x}^{2}-v_{0 x}^{2}\right| d x\right),
\end{aligned}
$$


where $t \in\left[0, T_{0}\right)$, functions $u$ and $v$ are two local or global strong solutions of problem (2) with initial data $u(0, \cdot)=u_{0} \epsilon$ $H^{1}(R)$ and $v(0, \cdot)=v_{0} \in H^{1}(R)$, respectively. Constant $c$ depends on $\left\|u_{0}\right\|_{H^{1}(R)},\left\|v_{0}\right\|_{H^{1}(R)}$, and the maximum existence time $T_{0}$. Secondly, from (5), we immediately arrive at the goal of the uniqueness. Here we state that the approach to establish (5) is the device of doubling variables which was presented in Kruzkov's paper [17].

This paper is organized as follows. Several lemmas are given in Section 2, while the proofs of the main results are established in Section 3.

\section{Notations and Several Lemmas}

Set $\xi_{T}=[0, T] \times R$ for an arbitrary $T>0$. The space of all infinitely differentiable functions $f(t, x)$ with compact support in $[0, T] \times R$ is denoted by $C_{0}^{\infty}\left(\xi_{T}\right)$. We define $\rho(\sigma)$ as a function which is infinitely differentiable on $(-\infty,+\infty)$ such that $\rho(\sigma) \geq 0, \rho(\sigma)=0$ for $|\sigma| \geq 1$ and $\int_{-\infty}^{\infty} \rho(\sigma) d \sigma=1$. For any number $h>0$, we let $\rho_{h}(\sigma)=\rho\left(h^{-1} \sigma\right) / h$. Then we have that $\rho_{h}(\sigma)$ is a function in $C^{\infty}(-\infty, \infty)$ and

$$
\begin{gathered}
\rho_{h}(\sigma) \geq 0, \quad \rho_{h}(\sigma)=0 \quad \text { if }|\sigma| \geq h, \\
\left|\rho_{h}(\sigma)\right| \leq \frac{c}{h}, \quad \int_{-\infty}^{\infty} \rho_{h}(\sigma)=1 .
\end{gathered}
$$

Assume that the function $v(x)$ is locally integrable in $(-\infty$, $\infty)$. We define the approximation of function $v(x)$ as

$$
v^{h}(x)=\frac{1}{h} \int_{-\infty}^{\infty} \rho\left(\frac{x-y}{h}\right) v(y) d y, \quad h>0 .
$$

We call $x_{0}$ a Lebesgue point of function $v(x)$ if

$$
\lim _{h \rightarrow 0} \frac{1}{h} \int_{\left|x-x_{0}\right| \leq h}\left|v(x)-v\left(x_{0}\right)\right| d x=0 .
$$

At any Lebesgue point $x_{0}$ of the function $v(x)$, we have $\lim _{h \rightarrow 0} v^{h}\left(x_{0}\right)=v\left(x_{0}\right)$. Since the set of points which are not Lebesgue points of $v(x)$ has measured zero, we get $v^{h}(x) \rightarrow$ $v(x)$ as $h \rightarrow 0$ almost everywhere.

We introduce notation connected with the concept of a characteristic cone. For any $M_{0}>0$, we define $N>$ $\sup _{t \in[0, \infty)}\|u\|_{L^{\infty}(R)}<\infty$. Let $\mho \delta$ designate the cone $\{(t, x)$ : $\left.|x| \leq M_{0}-N t, 0<t<T_{0}=\min \left(\mathrm{T}, M_{0} N^{-1}\right)\right\}$. We let $S_{\tau}$ designate the cross section of the cone $\mho$ by the plane $t=$ $\tau, \tau \in\left[0, T_{0}\right]$.

Let $H_{r}=\{x:|x| \leq r\}$, where $r>0$.

Lemma 1 (see [17]). Let the function $v(t, x)$ be bounded and measurable in cylinder $\Omega=[0, T] \times H_{r}$. If $\delta \in(0, \min [r, T])$ and any number $h \in(0, \delta)$, then the function

$$
V_{h}=\frac{1}{h^{2}} \iiint \int_{\substack{|(t-\tau) / 2| \leq h, \delta \leq(t+\tau) / 2 \leq T-\delta,|(x-y) / 2| \leq h,|(x+y) / 2| \leq r-\delta}}|v(t, x)-v(\tau, y)| d x d t d y d \tau
$$

satisfies $\lim _{h \rightarrow 0} V_{h}=0$.
Lemma 2 (see [17]). If the function $|\partial F(u) / \partial u|$ is bounded, then the function $H(u, v)=\operatorname{sign}(u-v)(F(u)-F(v))$ satisfies the Lipschitz condition in $u$ and $v$, respectively.

Lemma 3. Let $u_{0} \in H^{1}(R)$. It holds that the function $Q_{u}(t$, $x)=\partial_{x} P_{u}(t, x)=\partial_{x} \Lambda^{-2}\left(u^{2}+(1 / 2) u_{x}^{2}\right)$ satisfies

$$
\begin{gathered}
\left\|P_{u}(t, x)\right\|_{L^{\infty}(R)}<\infty, \\
\left\|P_{u}(t, x)\right\|_{L^{1}(R)}<\infty, \\
\left\|P_{u}(t, x)\right\|_{L^{2}(R)}<\infty, \\
\left\|Q_{u}(t, x)\right\|_{L^{\infty}(R)}<\infty, \\
\left\|Q_{u}(t, x)\right\|_{L^{1}(R)}<\infty, \\
\left\|Q_{u}(t, x)\right\|_{L^{2}(R)}<\infty .
\end{gathered}
$$

The proof of Lemma 3 can be found in $[13,15]$ (see [13, Lemma 5.1]).

Lemma 4. Let $u$ be the strong solution of problem $(2), f(t, x) \in$ $C_{0}^{\infty}\left(\xi_{T}\right)$, and $f(0, x)=0$. Then

$$
\begin{gathered}
\iint_{\xi_{T}}\left\{|u-k| f_{t}+\operatorname{sign}(u-k) \frac{1}{2}\left[u^{2}-k^{2}\right] f_{x}\right. \\
\left.-\operatorname{sign}(u-k) Q_{u}(t, x) f\right\} d x d t=0,
\end{gathered}
$$

where $k$ is an arbitrary constant.

Proof. Let $\Phi(u)$ be an arbitrary twice smooth function on the line $-\infty<u<\infty$. We multiply (2) by the function $\Phi^{\prime}(u) f(t, x)$, where $f(t, x) \in C_{0}^{\infty}\left(\xi_{T}\right)$. Integrating over $\xi_{T}$ and transferring the derivatives with respect to $t$ and $x$ to the test function $f$, for any constant $k$, we obtain

$$
\begin{array}{r}
\iint_{\xi_{T}}\left\{\Phi(u) f_{t}+\left[\int_{k}^{u} \Phi^{\prime}(z) z d z\right] f_{x}\right. \\
\left.-\Phi^{\prime}(u) Q_{u}(t, x) f\right\} d x d t=0
\end{array}
$$

in which we have used $\int_{-\infty}^{\infty}\left[\int_{k}^{u} \Phi^{\prime}(z) z d z\right] f_{x} d x=$ $-\int_{-\infty}^{\infty}\left[f \Phi^{\prime}(u) u u_{x}\right] d x$.

We have

$$
\begin{aligned}
\int_{-\infty}^{\infty}\left[\int_{k}^{u} \Phi^{\prime}(z) z d z\right] f_{x} d x \\
=\int_{-\infty}^{\infty}\left[\Phi^{\prime}(u)\left(\frac{1}{2} u^{2}-\frac{1}{2} k^{2}\right)\right. \\
\left.\quad-\frac{1}{2} \int_{k}^{u}\left(z^{2}-k^{2}\right) \Phi^{\prime \prime}(z) d z\right] f_{x} d x .
\end{aligned}
$$


Let $\Phi^{h}(u)$ be an approximation of the function $|u-k|$ and set $\Phi(u)=\Phi^{h}(u)$. Using the properties of the $\operatorname{sign}(u-k),(12)$, and (13) and sending $h \rightarrow 0$, we have

$$
\begin{gathered}
\iint_{\xi_{T}}\left\{|u-k| f_{t}+\operatorname{sign}(u-k) \frac{1}{2}\left[u^{2}-k^{2}\right] f_{x}\right. \\
\left.-\operatorname{sign}(u-k) Q_{u}(t, x) f\right\} d x d t=0,
\end{gathered}
$$

which completes the proof.

In fact, the proof of (11) can also be found in [17].

Lemma 5. Assume u and $v$ are two strong solutions of problem (2). It has

$$
\begin{aligned}
& \left|\int_{-\infty}^{\infty} \operatorname{sign}(u-v)\left[Q_{u}(t, x)-Q_{v}(t, x)\right] f d x\right| \\
& \quad \leq c \int_{-\infty}^{\infty}\left(\left|u_{0}^{2}-v_{0}^{2}\right|+\left|u_{0 x}^{2}-v_{0 x}^{2}\right|+|u-v|\right) d x .
\end{aligned}
$$

Proof. We have

$$
\begin{aligned}
\int_{-\infty}^{\infty}\left(u_{x}^{2}-v_{x}^{2}\right) d x=\int_{-\infty}^{\infty}\left[\left(u_{0}^{2}+u_{0 x}^{2}\right)-\left(v_{0}^{2}+v_{0 x}^{2}\right)\right] d x \\
\mid \int_{-\infty}^{\infty} \quad \Lambda_{-\infty}^{-2}\left(u^{2}+\frac{1}{2} u_{x}^{2}-v^{2}-\frac{1}{2} v_{x}^{2}\right) \\
\quad \times \partial_{x}[\operatorname{sign}[u(t, x)-v(t, x)] f(t, x)] d x \mid \\
=\mid \frac{1}{2} \iint_{-\infty}^{\infty} e^{-|x-y|}\left(u^{2}+\frac{1}{2} u_{y}^{2}-v^{2}-\frac{1}{2} v_{y}^{2}\right) d y \\
\quad \times \partial_{x}[\operatorname{sign}[u(t, x)-v(t, x)] f(t, x)] d x \mid \\
\leq \frac{1}{2} \int_{-\infty}^{\infty}\left|\int_{-\infty}^{\infty}\left(u^{2}+\frac{1}{2} u_{y}^{2}-v^{2}-\frac{1}{2} v_{y}^{2}\right) d y\right| \\
\quad \times \int_{-\infty}^{\infty}\left|\int_{x}[\operatorname{sign}[u(t, x)-v(t, x)] f(t, x)]\right| d x \\
\quad \frac{1}{2} \mid \int_{-\infty}^{\infty}\left(\partial_{x}[\operatorname{sign}[u(t, x)-v(t, x)] f(t, x)] \mid d x\right.
\end{aligned}
$$

$$
\begin{gathered}
\leq c\left(\int_{-\infty}^{\infty}\left|u_{0}^{2}-v_{0}^{2}\right| d x+\int_{-\infty}^{\infty}\left|u_{0 x}^{2}-v_{0 x}^{2}\right| d x\right. \\
\left.+\int_{-\infty}^{\infty}|u-v| d x\right),
\end{gathered}
$$

in which we have used the Fubini theorem, $\|u\|_{L^{\infty}} \leq\left\|u_{0}\right\|_{H^{1}(R)}$ and $\|v\|_{L^{\infty}} \leq\left\|v_{0}\right\|_{H^{1}(R)}$. The proof is completed.

\section{Main Results}

Theorem 6. Let $u$ and $v$ be two local or global strong solutions of problem (2) with initial data $u(0, \cdot)=u_{0} \in H^{1}(R)$ and $v(0, \cdot)=v_{0} \in H^{1}(R)$, respectively. Let $T_{0}$ be the maximum existence time of solutions $u$ and $v$. For any $t \in\left[0, T_{0}\right)$, it holds that

$$
\begin{aligned}
& \|u(t, \cdot)-v(t, \cdot)\|_{L^{1}(R)} \\
& \leq e^{c t} c\left(1+T_{0}\right) \\
& \quad \times\left(\int_{-\infty}^{\infty}\left|u_{0}(x)-v_{0}(x)\right| d x+\int_{-\infty}^{\infty}\left|u_{0 x}^{2}-v_{0 x}^{2}\right| d x\right),
\end{aligned}
$$

where $c$ depends on $\left\|u_{0}\right\|_{H^{1}(R)}$ and $\left\|v_{0}\right\|_{H^{1}(R)}$.

From Theorem 6, we immediately obtain the uniqueness result.

Theorem 7. Let $u(t, x)$ be a strong solution of (1) with $u_{0} \in$ $H^{1}(R)$, and let $T_{0}$ be the maximum existence time of solution $u$. Then any strong solution of (1) is unique.

Proof of Theorem 6. For an arbitrary $T>0$, set $\xi_{T}=[0, T] \times R$. Let $f(t, x) \in C_{0}^{\infty}\left(\xi_{T}\right)$. We assume that $f(t, x)=0$ outside some cylinder

$$
\biguplus=\{(t, x)\}=[\delta, T-2 \delta] \times H_{r-2 \delta}, \quad 0<2 \delta \leq \min (T, r) .
$$

We define

$$
\begin{aligned}
g & =f\left(\frac{t+\tau}{2}, \frac{x+y}{2}\right) \rho_{h}\left(\frac{t-\tau}{2}\right) \rho_{h}\left(\frac{x-y}{2}\right) \\
& =f(\cdots) \lambda_{h}(*)
\end{aligned}
$$

where $(\cdots)=((t+\tau) / 2,(x+y) / 2)$ and $(*)=((t-\tau) / 2,(x-$ $y) / 2$ ). The function $\rho(\sigma)$ is defined in (6). Note that

$$
\begin{aligned}
g_{t}+g_{\tau} & =f_{t}(\cdots) \lambda_{h}(*), \\
g_{x}+g_{y} & =f_{x}(\cdots) \lambda_{h}(*)
\end{aligned}
$$


Taking $k=v(\tau, y)$ and assuming $f(t, x)=0$ outside the cylinder $\biguplus$, from Lemma 4 , we have

$$
\begin{aligned}
\iiint \int_{\xi_{T} \times \xi_{T}}\{ & |u(t, x)-v(\tau, y)| g_{t}+\operatorname{sign}(u(t, x)-v(\tau, y)) \\
& \times\left(\frac{u^{2}(t, x)}{2}-\frac{v^{2}(\tau, y)}{2}\right) g_{x} \\
& +\operatorname{sign}(u(t, x)-v(\tau, y)) \\
& \left.\times Q_{u}(t, x) g\right\} d x d t d y d \tau=0 .
\end{aligned}
$$

Similarly, it has

$$
\begin{aligned}
\iiint \int_{\xi_{T} \times \xi_{T}}\{\mid & \left|\tau(\tau, y)-u(t, x) g_{\tau}\right|+\operatorname{sign}(v(\tau, y)-u(t, x)) \\
& \times\left(\frac{v^{2}(\tau, y)}{2}-\frac{u^{2}(t, x)}{2}\right) g_{y} \\
& +\operatorname{sign}(v(\tau, y)-u(t, x)) \\
& \left.\times Q_{v}(\tau, y) g\right\} d x d t d y d \tau=0
\end{aligned}
$$

from which we obtain

$$
\begin{aligned}
& 0 \leq \iiint \int_{\xi_{T} \times \xi_{T}}\left\{|u(t, x)-v(\tau, y)|\left(g_{t}+g_{\tau}\right)\right. \\
& +\operatorname{sign}(u(t, x)-v(\tau, y)) \\
& \times\left(\frac{u^{2}(t, x)}{2}-\frac{v^{2}(\tau, y)}{2}\right) \\
& \left.\times\left(g_{x}+g_{y}\right)\right\} d x d t d y d \tau \\
& +\mid \iiint \int_{\xi_{T} \times \xi_{T}} \operatorname{sign}(u(t, x)-v(t, x)) \\
& \times\left(Q_{u}(t, x)-Q_{v}(\tau, y)\right) g d x d t d y d \tau \mid \\
& =\iiint \int_{\xi_{T} \times \xi_{T}}\left(I_{1}+I_{2}+I_{3}\right) d x d t d y d \tau .
\end{aligned}
$$

We will show that

$$
\begin{gathered}
0 \leq \iint_{\xi_{T}}\left\{|u(t, x)-v(t, x)| f_{t}+\operatorname{sign}(u(t, x)-v(t, x))\right. \\
\left.\times\left(\frac{u^{2}(t, x)}{2}-\frac{v^{2}(t, x)}{2}\right) f_{x}\right\} d x d t
\end{gathered}
$$

$$
\begin{aligned}
& +\mid \iint_{\xi_{T}} \operatorname{sign}(u(t, x)-v(t, x)) \\
& \quad \times\left[Q_{u}(t, x)-Q_{v}(t, x)\right] f d x d t \mid .
\end{aligned}
$$

We note that the first two terms in the integrand of (23) can be represented in the form

$$
Y_{h}=Y(t, x, \tau, y, u(t, x), v(\tau, y)) \lambda_{h}(*) .
$$

Since $\|u\|_{L^{\infty}} \leq\left\|u_{0}\right\|_{H^{1}(R)}$ and $\|v\|_{L^{\infty}} \leq\left\|v_{0}\right\|_{H^{1}(R)}$, from Lemma 2, we know $Y_{h}$ satisfies the Lipschitz condition in $u$ and $v$, respectively. By the choice of $g$, we have $Y_{h}=0$ outside the region

$$
\begin{aligned}
& \{(t, x ; \tau, y)\}=\left\{\delta \leq \frac{t+\tau}{2} \leq T-2 \delta, \frac{|t-\tau|}{2} \leq h,\right. \\
& \left.\frac{|x+y|}{2} \leq r-2 \delta, \frac{|x-y|}{2} \leq h\right\}, \\
& \iiint \int_{\xi_{T} \times \xi_{T}} Y_{h} d x d t d y d \tau \\
& =\iiint \int_{\xi_{T} \times \xi_{T}}[Y(t, x, \tau, y, u(t, x), v(\tau, y)) \\
& \quad \times \lambda_{h}(*) d x d t d y d \tau \\
& \quad+\iiint_{\xi_{T} \times \xi_{T}} Y(t, x, t, x, u(t, x), v(t, x)) \\
& \quad \times \lambda_{h}(*) d x d t d y d \tau \\
& =J_{1}(h)+J_{2} .
\end{aligned}
$$

Considering the estimate $|\lambda(*)| \leq c / h^{2}$ and the expression of function $Y_{h}$, we have

$$
\begin{aligned}
& \left|J_{1}(h)\right| \\
& \leq c\left[h+\frac{1}{h^{2}}\right.
\end{aligned}
$$

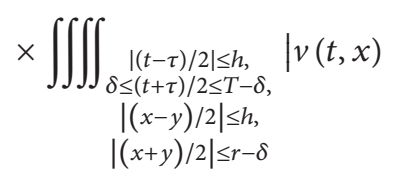$$
-v(\tau, y) \mid d x d t d y d \tau,
$$ 
where the constant $c$ does not depend on $h$. Using Lemma 1 , we obtain $J_{1}(h) \rightarrow 0$ as $h \rightarrow 0$. The integral $J_{2}$ does not depend on $h$. In fact, substituting $t=\alpha,(t-\tau) / 2=\beta, x=\eta$, $(x-y) / 2=\xi$ and noting that

$$
\int_{-h}^{h} \int_{-\infty}^{\infty} \lambda_{h}(\beta, \xi) d \xi d \beta=1
$$

we have

$$
\begin{aligned}
J_{2}= & 2^{2} \iint_{\xi_{T}} Y_{h}(\alpha, \eta, \alpha, \eta, u(\alpha, \eta), v(\alpha, \eta)) \\
& \times\left\{\int_{-h}^{h} \int_{-\infty}^{\infty} \lambda_{h}(\beta, \xi) d \xi d \beta\right\} d \eta d \alpha \\
= & 4 \iint_{\xi_{T}} Y_{h}(t, x, t, x, u(t, x), v(t, x)) d x d t .
\end{aligned}
$$

Hence

$$
\begin{aligned}
& \lim _{h \rightarrow 0} \iiint \int_{\xi_{T} \times \xi_{T}} Y_{h} d x d t d y d \tau \\
& \quad=4 \iint_{\xi_{T}} Y_{h}(t, x, t, x, u(t, x), v(t, x)) d x d t .
\end{aligned}
$$

Since

$$
\begin{gathered}
I_{3}=\operatorname{sign}(u(t, x)-v(\tau, y))\left(Q_{u}(t, x)-Q_{v}(\tau, y)\right) f \lambda_{h}(*), \\
\iiint \int_{\xi_{T} \times \xi_{T}} I_{3} d x d t d y d \tau \\
=\iiint \int_{\xi_{T} \times \xi_{T}}\left[I_{3}(t, x, \tau, y)-I_{3}(t, x, t, x)\right] \\
\quad \times \lambda_{h}(*) d x d t d y d \tau \\
\quad+\iiint \int_{\xi_{T} \times \xi_{T}} I_{3}(t, x, t, x) \lambda_{h}(*) d x d t d y d \tau \\
=K_{1}(h)+K_{2},
\end{gathered}
$$

we obtain

$\left|K_{1}(h)\right|$

$$
\begin{aligned}
& \leq c\left(h+\frac{1}{h^{2}}\right. \\
& \times \iiint \int_{\substack{\delta \leq(t+\tau) / 2 \leq T-\delta,|(x-y) / 2| \leq h,|(x+y) / 2| \leq r-\delta}} \mid Q_{v}(t, x) \\
& \left.-Q_{v}(\tau, y) \mid d x d t d y d \tau\right) \text {. }
\end{aligned}
$$

By Lemmas 1 and 3, we have $K_{1}(h) \rightarrow 0$ as $h \rightarrow 0$. Using (28), we have

$$
\begin{aligned}
K_{2}=2^{2} \iint_{\xi_{T}} I_{3}(\alpha, \eta, \alpha, \eta, u(\alpha, \eta), v(\alpha, \eta)) \\
\quad \times\left\{\int_{-h}^{h} \int_{-\infty}^{\infty} \lambda_{h}(\beta, \xi) d \xi d \beta\right\} d \eta d \alpha \\
=4 \iint_{\xi_{T}} I_{3}(t, x, t, x, u(t, x), v(t, x)) d x d t \\
=4 \iint_{\xi_{T}} \operatorname{sign}(u(t, x)-v(t, x)) \\
\quad \times\left(Q_{u}(t, x)-Q_{v}(t, x)\right) f(t, x) d x d t .
\end{aligned}
$$

From (30) and (33), we prove that inequality (24) holds.

Let

$$
\omega(t)=\int_{-\infty}^{\infty}|u(t, x)-v(t, x)| d x .
$$

We define

$$
\theta_{h}=\int_{-\infty}^{\sigma} \rho_{h}(\sigma) d \sigma \quad\left(\theta_{h}^{\prime}(\sigma)=\rho_{h}(\sigma) \geq 0\right)
$$

and choose two numbers $\tau_{1}$ and $\tau_{2} \in\left(0, T_{0}\right), \tau_{1}<\tau_{2}$. In (24), we choose

$$
\begin{array}{r}
f=\left[\theta_{h}\left(t-\tau_{1}\right)-\theta_{h}\left(t-\tau_{2}\right)\right] \chi(t, x), \\
h<\min \left(\tau_{1}, T_{0}-\tau_{2}\right),
\end{array}
$$

where

$$
\begin{aligned}
\chi(t, x) & =\chi_{\varepsilon}(t, x) \\
& =1-\theta_{\varepsilon}\left(|x|+N t-M_{0}+\varepsilon\right), \quad \varepsilon>0 .
\end{aligned}
$$

We note that function $\chi(t, x)=0$ outside the cone $\mho$ and $f(t, x)=0$ outside the set $\biguplus$. For $(t, x) \in \mho$, we have the relations

$$
0=\chi_{t}+N\left|\chi_{x}\right| \geq \chi_{t}+N \chi_{x} .
$$

Applying (24) and (35)-(38), we have the inequality

$$
\begin{aligned}
& 0 \leq \iint_{\xi_{T_{0}}}\{ {\left[\rho_{h}\left(t-\tau_{1}\right)-\rho_{h}\left(t-\tau_{2}\right)\right] } \\
&\left.\quad \times \chi_{\varepsilon}|u(t, x)-v(t, x)|\right\} d x d t \\
&+\mid \iint_{\xi_{T_{0}}}\left[\theta_{h}\left(t-\tau_{1}\right)-\theta_{h}\left(t-\tau_{2}\right)\right] \\
& \quad \times\left[Q_{u}(t, x)-Q_{v}(t, x)\right] B(t, x) \chi(t, x) d x d t \mid,
\end{aligned}
$$

where $B(t, x)=\operatorname{sign}[u(t, x)-v(t, x)]$. 
From (39), we obtain

$$
\begin{aligned}
0 \leq \iint_{\xi_{T_{0}}}\left\{\left[\rho_{h}\left(t-\tau_{1}\right)-\rho_{h}\left(t-\tau_{2}\right)\right]\right. \\
\left.\quad \times \chi_{\varepsilon}|u(t, x)-v(t, x)|\right\} d x d t \\
\quad+\int_{0}^{T_{0}}\left(\theta_{h}\left(t-\tau_{1}\right)-\theta_{h}\left(t-\tau_{2}\right)\right) \\
\quad \times\left|\int_{-\infty}^{\infty}\left[Q_{u}(t, x)-Q_{v}(t, x)\right] B(t, x) \chi(t, x) d x\right| d t .
\end{aligned}
$$

Using Lemma 5, we have

$$
\begin{aligned}
& 0 \leq \iint_{\xi_{T_{0}}}\left\{\left[\rho_{h}\left(t-\tau_{1}\right)-\rho_{h}\left(t-\tau_{2}\right)\right]\right. \\
&\left.\times \chi_{\varepsilon}|u(t, x)-v(t, x)|\right\} d x d t \\
&+c \int_{0}^{T_{0}}\left(\theta_{h}\left(t-\tau_{1}\right)-\theta_{h}\left(t-\tau_{2}\right)\right) \\
& \quad \times\left(G+\int_{-\infty}^{\infty}|u-v| d x\right) d t
\end{aligned}
$$
where $G=\int_{-\infty}^{\infty}\left|u_{0}^{2}-v_{0}^{2}\right| d x+\int_{-\infty}^{\infty}\left|u_{0 x}^{2}-v_{0 x}^{2}\right| d x$ and $c$ is defined
in Lemma 5.

Letting $\varepsilon \rightarrow 0$ in (41) and sending $M_{0} \rightarrow \infty$, we have

$$
\begin{aligned}
0 \leq \int_{0}^{T_{0}}\{ & {\left[\rho_{h}\left(t-\tau_{1}\right)-\rho_{h}\left(t-\tau_{2}\right)\right] } \\
& \left.\times \int_{-\infty}^{\infty}|u(t, x)-v(t, x)| d x\right\} d t \\
+c \int_{0}^{T_{0}}\left(\theta_{h}\left(t-\tau_{1}\right)-\theta_{h}\left(t-\tau_{2}\right)\right) & \\
& \times\left(G+\int_{-\infty}^{\infty}|u-v| d x\right) d t
\end{aligned}
$$

By the properties of the function $\rho_{h}(\sigma)$ for $h \leq \min \left(\tau_{1}\right.$, $\left.T_{0}-\tau_{1}\right)$, we have

$$
\begin{aligned}
& \left|\int_{0}^{T_{0}} \rho_{h}\left(t-\tau_{1}\right) \omega(t) d t-\omega\left(\tau_{1}\right)\right| \\
& \quad=\left|\int_{0}^{T_{0}} \rho_{h}\left(t-\tau_{1}\right)\left[\omega(t)-\omega\left(\tau_{1}\right)\right] d t\right| \\
& \quad \leq c \frac{1}{h} \int_{\tau_{1}-h}^{\tau_{1}+h}\left|\omega(t)-\omega\left(\tau_{1}\right)\right| d t \longrightarrow 0 \text { as } h \longrightarrow 0,
\end{aligned}
$$

where $c$ is independent of $h$.

Set

$$
L\left(\tau_{1}\right)=\int_{0}^{T_{0}} \theta_{h}\left(t-\tau_{1}\right) \mu(t) d t=\int_{0}^{T_{0}} \int_{-\infty}^{t-\tau_{1}} \rho_{h}(\sigma) d \sigma \omega(t) d t .
$$

Using the similar proof of (43), we get

$$
L^{\prime}\left(\tau_{1}\right)=-\int_{0}^{T_{0}} \rho_{h}\left(t-\tau_{1}\right) \omega(t) d t \longrightarrow-\omega\left(\tau_{1}\right) \quad \text { as } h \longrightarrow 0,
$$

from which we obtain

$$
L\left(\tau_{1}\right) \longrightarrow L(0)-\int_{0}^{\tau_{1}} \omega(\sigma) d \sigma \quad \text { as } h \longrightarrow 0 .
$$

Similarly, we have

$$
L\left(\tau_{2}\right) \longrightarrow L(0)-\int_{0}^{\tau_{2}} \omega(\sigma) d \sigma \quad \text { as } h \longrightarrow 0 .
$$

Then, we get

$$
L\left(\tau_{1}\right)-L\left(\tau_{2}\right) \longrightarrow \int_{\tau_{1}}^{\tau_{2}} \omega(\sigma) d \sigma \quad \text { as } h \longrightarrow 0 .
$$

Furthermore, if $h \rightarrow 0$, we have

$$
\begin{aligned}
& \int_{0}^{T_{0}}\left(\theta_{h}\left(t-\tau_{1}\right)-\theta_{h}\left(t-\tau_{2}\right)\right) G d t \rightarrow G \int_{\tau_{1}}^{\tau_{2}} d t \\
& \quad=\left(\int_{-\infty}^{\infty}\left|u_{0}^{2}-v_{0}^{2}\right| d x+\int_{-\infty}^{\infty}\left|u_{0 x}^{2}-v_{0 x}^{2}\right| d x\right)\left(\tau_{2}-\tau_{1}\right) .
\end{aligned}
$$

Let $\tau_{1} \rightarrow 0$ and $\tau_{2} \rightarrow t$, and note that

$$
\begin{aligned}
\left|u\left(\tau_{1}, x\right)-v\left(\tau_{1}, x\right)\right| \leq & \left|u\left(\tau_{1}, x\right)-u_{0}(x)\right| \\
& +\left|v\left(\tau_{1}, x\right)-v_{0}(x)\right| \\
& +\left|u_{0}(x)-v_{0}(x)\right| .
\end{aligned}
$$

Thus, from (42), (43), (48), (49), and (50), for any $t \in$ $\left[0, T_{0}\right]$, we have

$$
\begin{aligned}
& \int_{-\infty}^{\infty}|u(t, x)-v(t, x)| d x \\
& \quad \leq \int_{-\infty}^{\infty}|u(0, x)-v(0, x)| d x \\
& \quad+c T_{0}\left(\int_{-\infty}^{\infty}\left|u_{0}^{2}-v_{0}^{2}\right| d x+\int_{-\infty}^{\infty}\left|u_{0 x}^{2}-v_{0 x}^{2}\right| d x\right) \\
& \quad+\int_{0}^{t} \int_{-\infty}^{\infty}|u-v| d x
\end{aligned}
$$

from which we complete the proof of Theorem 6 by using the Gronwall inequality.

\section{Acknowledgments}

Thanks are given to referees whose suggestions are very helpful to the paper. This work is supported by both the Fundamental Research Funds for the Central Universities (JBK120504) and the Applied and Basic Project of Sichuan Province (2012JY0020). 


\section{References}

[1] R. Camassa and D. D. Holm, "An integrable shallow water equation with peaked solitons," Physical Review Letters, vol. 71, no. 11, pp. 1661-1664, 1993.

[2] A. Constantin and J. Escher, "Global weak solutions for a shallow water equation," Indiana University Mathematics Journal, vol. 47, no. 4, pp. 1527-1545, 1998.

[3] A. Constantin and L. Molinet, "Global weak solutions for a shallow water equation," Communications in Mathematical Physics, vol. 211, no. 1, pp. 45-61, 2000.

[4] A. Constantin and J. Escher, "Global existence and blow-up for a shallow water equation," Annali della Scuola Normale Superiore di Pisa. Classe di Scienze, vol. 26, no. 2, pp. 303-328, 1998.

[5] A. A. Himonas and G. Misiołek, "The Cauchy problem for an integrable shallow-water equation," Differential and Integral Equations, vol. 14, no. 7, pp. 821-831, 2001.

[6] S. Lai and Y. Wu, "The local well-posedness and existence of weak solutions for a generalized Camassa-Holm equation," Journal of Differential Equations, vol. 248, no. 8, pp. 2038-2063, 2010.

[7] Y. A. Li and P. J. Olver, "Well-posedness and blow-up solutions for an integrable nonlinearly dispersive model wave equation," Journal of Differential Equations, vol. 162, no. 1, pp. 27-63, 2000.

[8] G. Rodríguez-Blanco, "On the Cauchy problem for the Camassa-Holm equation," Nonlinear Analysis: Theory, Methods \& Applications, vol. 46, no. 3, pp. 309-327, 2001.

[9] A. Constantin and J. Escher, "Well-posedness, global existence, and blowup phenomena for a periodic quasi-linear hyperbolic equation," Communications on Pure and Applied Mathematics, vol. 51, no. 5, pp. 475-504, 1998.

[10] A. Constantin and J. Escher, "Wave breaking for nonlinear nonlocal shallow water equations," Acta Mathematica, vol. 181, no. 2, pp. 229-243, 1998.

[11] A. Constantin and J. Escher, "On the Cauchy problem for a family of quasilinear hyperbolic equations," Communications in Partial Differential Equations, vol. 23, no. 7-8, pp. 1449-1458, 1998.

[12] A. Bressan and A. Constantin, "Global conservative solutions of the Camassa-Holm equation," Archive for Rational Mechanics and Analysis, vol. 183, no. 2, pp. 215-239, 2007.

[13] G. M. Coclite, H. Holden, and K. H. Karlsen, "Global weak solutions to a generalized hyperelastic-rod wave equation," SIAM Journal on Mathematical Analysis, vol. 37, no. 4, pp. 10441069, 2005.

[14] H. Holden and X. Raynaud, "Global conservative solutions of the Camassa-Holm equation-a Lagrangian point of view," Communications in Partial Differential Equations, vol. 32, no. 10-12, pp. 1511-1549, 2007.

[15] Z. Xin and P. Zhang, "On the weak solutions to a shallow water equation," Communications on Pure and Applied Mathematics, vol. 53, no. 11, pp. 1411-1433, 2000.

[16] Z. Yin, "On the Cauchy problem for an integrable equation with peakon solutions," Illinois Journal of Mathematics, vol. 47, no. 3 , pp. 649-666, 2003.

[17] S. Kruzkov, "First order quasi-linear equations in several independent variables," Mathematics of the USSR-Sbornik, vol. 10, no. 2, pp. 217-243, 1970. 


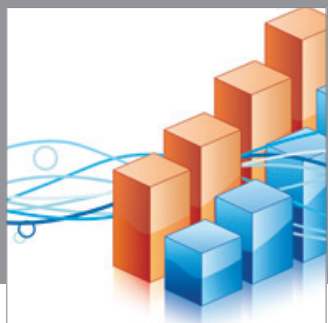

Advances in

Operations Research

mansans

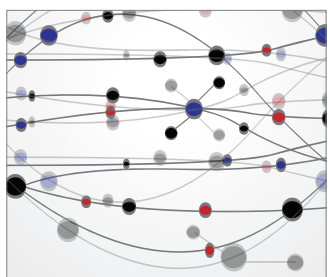

The Scientific World Journal
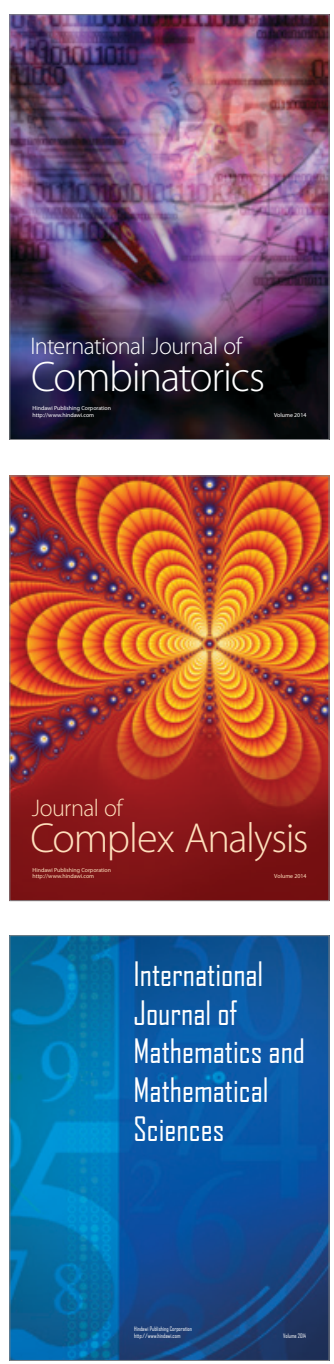
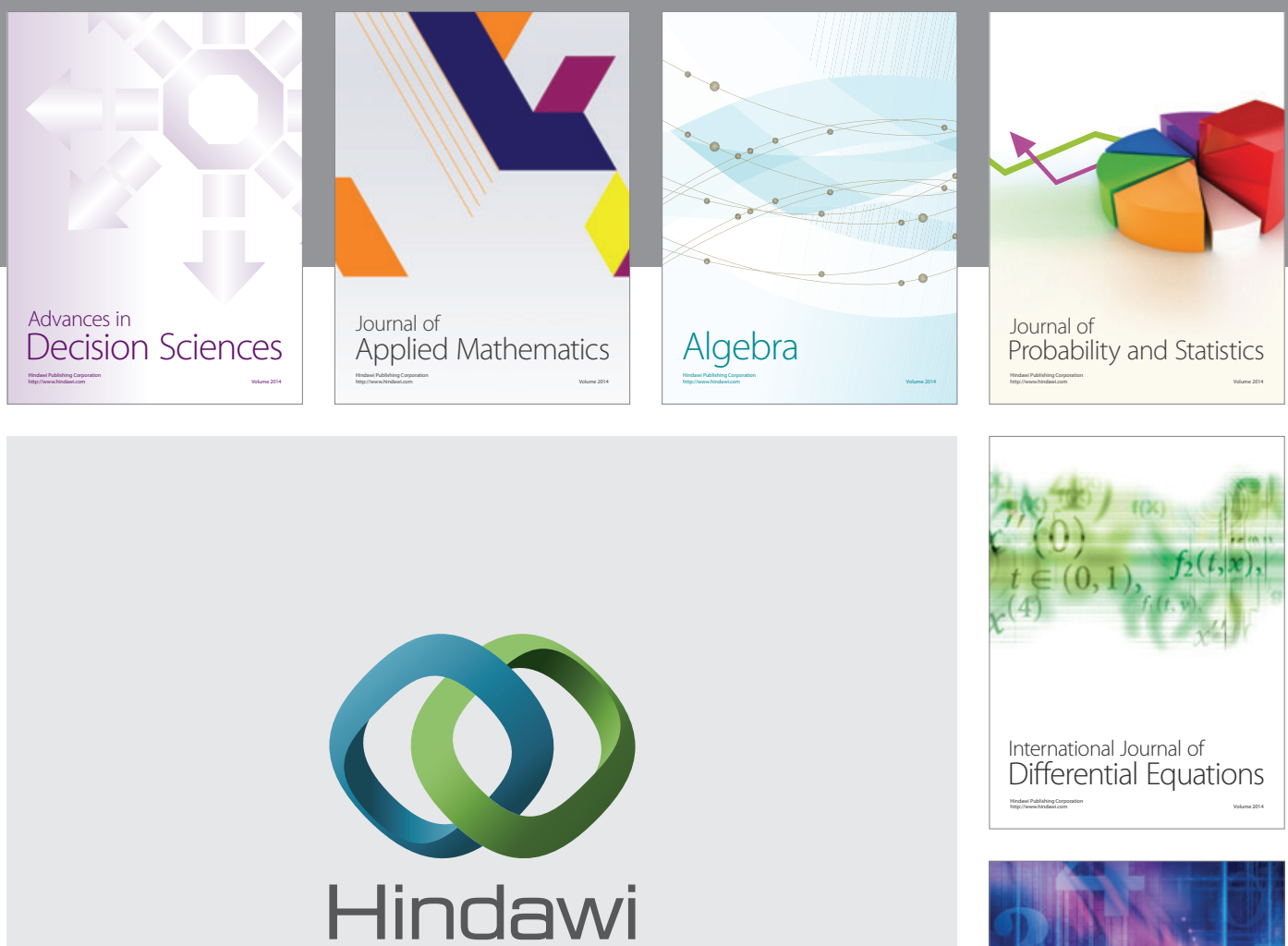

Submit your manuscripts at http://www.hindawi.com
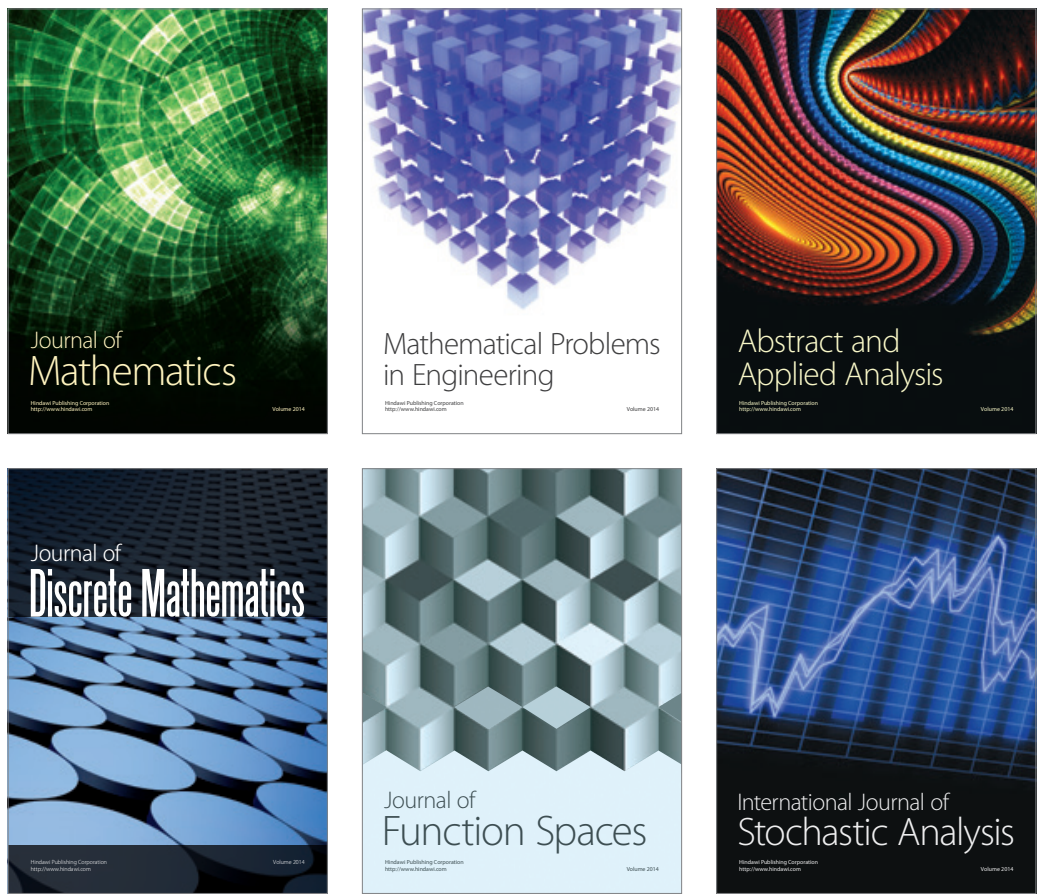

Journal of

Function Spaces

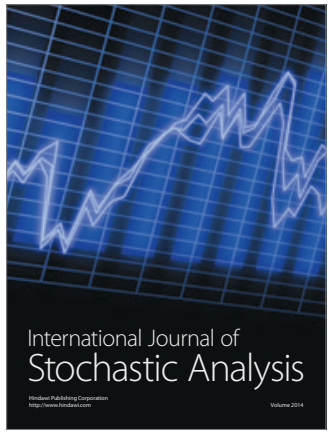

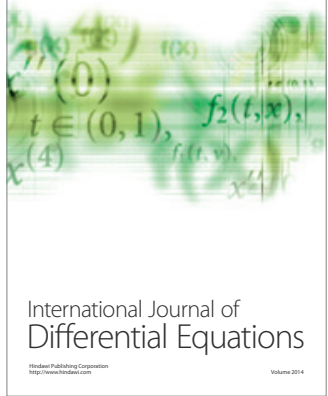
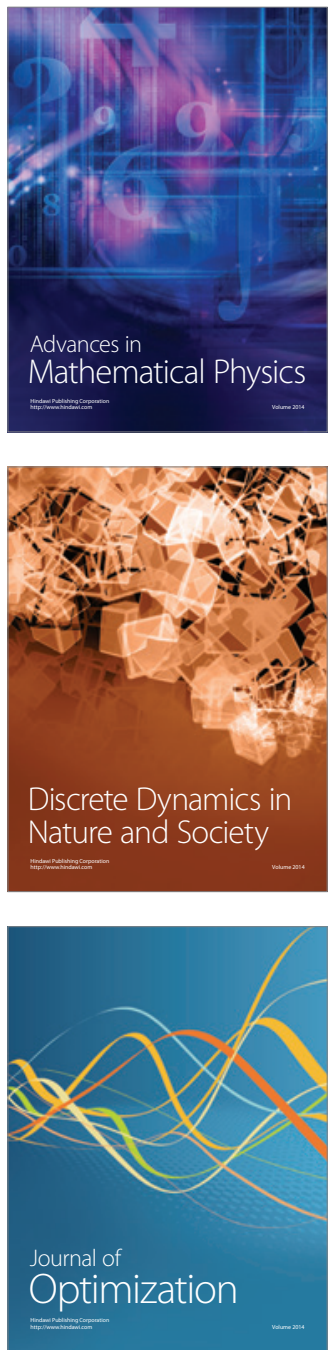